\title{
Myocardial infarction in severe haemophilia
}

Jenique Bailly, Johnny Mahlangu

With improved replacement therapy, people with haemophilia (PWH) live longer, with the consequent emergence of age-related chronic diseases not previously seen in haemophilia. The prevalence of cardiovascular disease and associated complications in older PWH appears to be on the rise. Management of myocardial infarction in a person living with severe haemophilia presents a particular challenge, as treatment options often include antiplatelet therapy, anti-coagulation and invasive procedures. Current guidelines for managing acute myocardial infarction are not specifically designed for people with bleeding diatheses such as haemophilia. We present a patient with severe haemophilia $A$ on extended half-life factor VIII prophylaxis who developed an acute ST-elevation myocardial infarction (STEMI), discuss his clinical management and highlight lessons learned.

Keywords: Coagulation disorders, Myocardial infarction, Severe haemophilia

JENIQUE BAILLY

Associate Lecturer, Department of Molecular Medicine and Haematology, University of the Witwatersrand, Johannesburg

\section{JOHNNY MAHLANGU}

Head of Clinical Haematology, Haemophilia

Comprehensive Care Centre, Charlotte Maxeke

Johannesburg Academic Hospital; Professor of

Haematology, Department of Molecular Medicine and

Haematology, University of the Witwatersrand and NHLS

Johannesburg. Email: johnny.mahlangu@nhls.ac.za

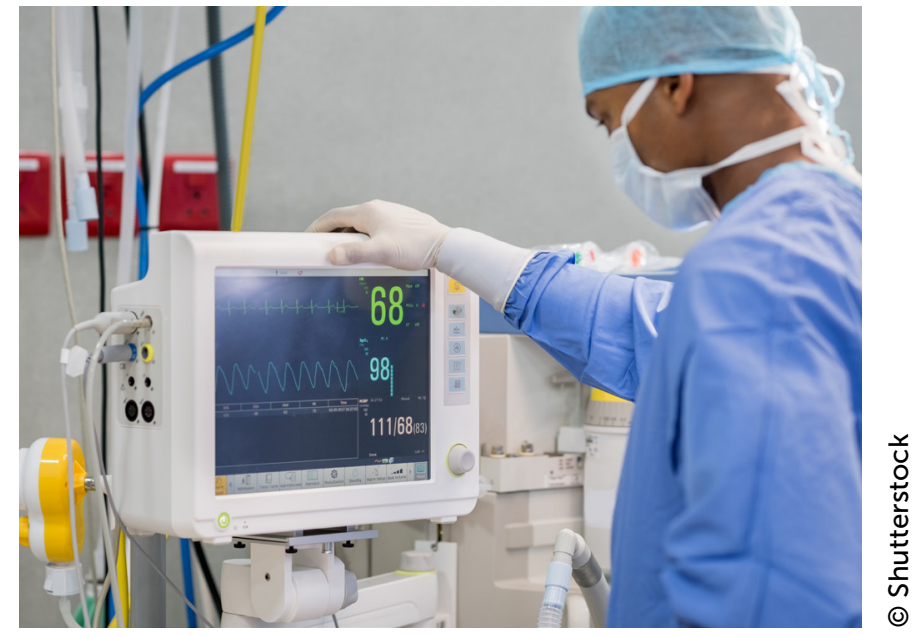

he recent development of therapies
with an improved safety, efficacy and
pharmacokinetic profile has resulted in
the enhanced survival of people with haemophilia (PWH). In many developed countries, their life expectancy is now similar to those without haemophilia. However, this improved longevity is accompanied by the emergence of age-related chronic disease not previously seen in the haemophilia population ${ }^{[1]}$. The risk of developing atherosclerosis and associated cardiovascular disease appears equivalent in $\mathrm{PWH}$ compared to age-matched males in the general population ${ }^{[1-4]}$. The emerging prevalence of cardiovascular disease in older $\mathrm{PWH}$ is reflected by the numerous case studies reported in recent years ${ }^{[5]}$. Management of myocardial infarction in a person living with severe haemophilia presents a particular challenge as treatment options often include antiplatelet therapy, anti-coagulation and invasive procedures. Here, we

This is an Open Access article distributed under the terms of the Creative Commons Attribution-NonCommercial-NoDerivs License (https://creativecommons.org/licenses/by-nc-nd/3.0/) which permits use and distribution in any medium, provided the original work is properly cited, the use is non-commercial, and no modifications or adaptations are made. Copyright is retained by the authors. 
present the case of a middle-aged male with severe haemophilia A on extended half-life clotting factor prophylaxis, with an acute ST elevation myocardial infarction (STEMI) requiring intervention.

\section{Case presentation}

This patient was a 54-year-old Caucasian male, who was diagnosed with severe haemophilia A shortly after birth. Intron 22 and intron 1 inversions were excluded; further analysis revealed a c.1336C $>$ T mutation in exon 9 of his $F 8$ gene. He was on extended half-life factor VIII (rFVIIIFc) prophylaxis at 3500 units (50 units/kg) every third day. He had no inhibitor and was bleedfree for at least four years prior to this event. As a child he was treated with HIV-infected plasma-derived product through which he acquired HIV infection. He was on anti-retroviral therapy and virologically suppressed with an absolute CD4 count of 750 cells/ $\mu \mathrm{L}$ and HIV viral load below detection level at the time of the event.
The patient presented to the Emergency Department in June 2016 with acute chest pain suggestive of an ischaemic cardiac event. An electrocardiogram (ECG) revealed ST elevation in leads V1-V3 as well as in lead AVL (see figure 1). Significant $q$-waves in the antero-lateral leads was interpreted as evidence of a previous myocardial infarct. Troponins and creatine kinase MB mass (CK-MB) at presentation were raised at $n=>50000 \mathrm{ng} / \mathrm{L}$ and $\mathrm{n}=>268 \mu \mathrm{g} / \mathrm{L}$ respectively. An echocardiograph revealed anterior wall hypokinesia with a left ventricular ejection fraction of $45 \%$. His factor VIII (FVIII) level at presentation was $69 \mathrm{IU} / \mathrm{dL}$ and he did not have FVIII inhibitors $(0,0 \mathrm{BU} / \mathrm{mL})$. His full blood count, renaland liver function tests were within normal limits. A diagnosis of acute STEMI was made.

The patient's cardio-vascular risk factors included male gender, being a heavy smoker (a 43-pack year smoking history), raised low density lipoprotein (LDL) and HIV infection. Although the latter was well

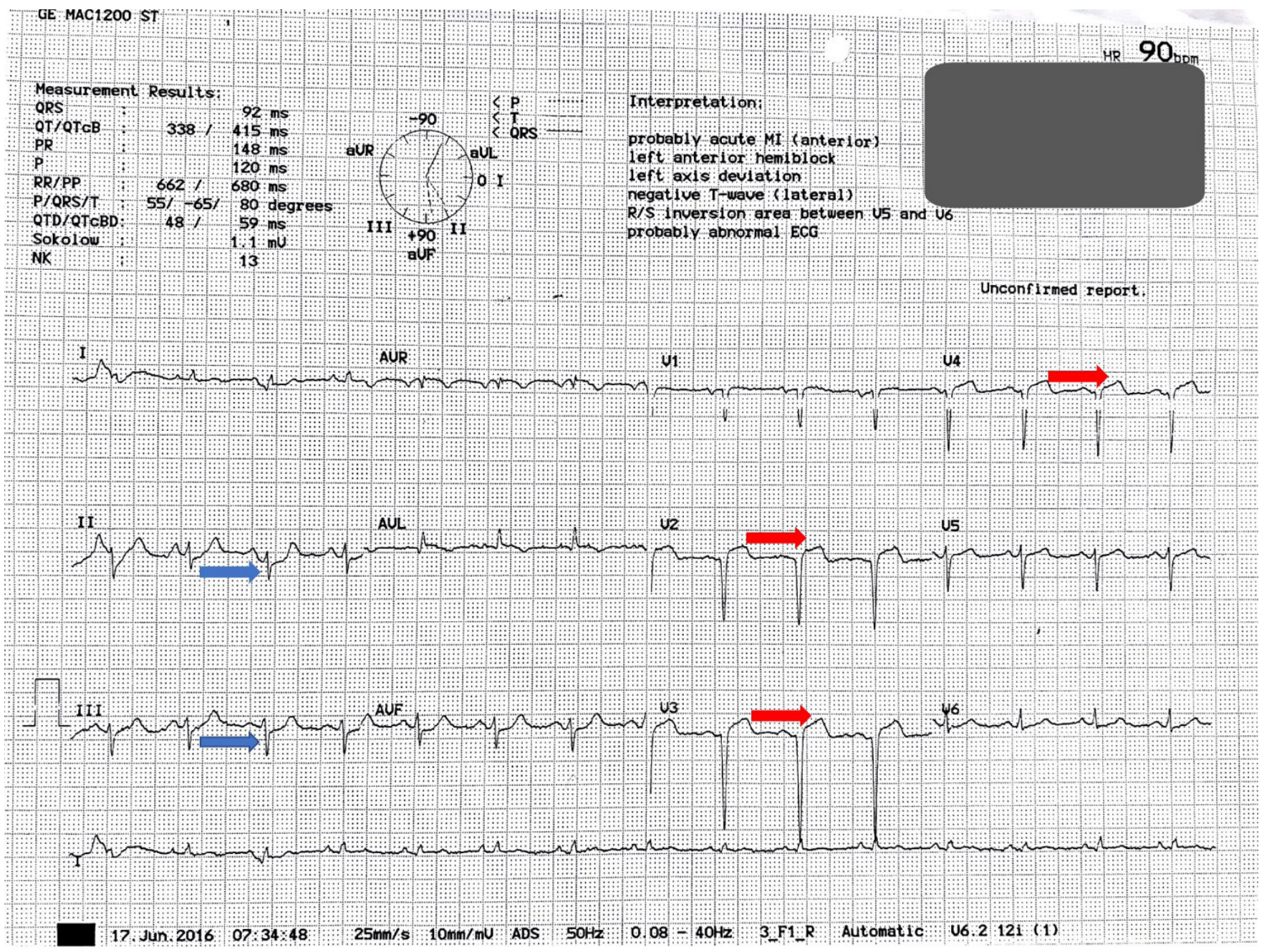

Figure 1: Electrocardiogram showing ST segment elevation in leads V2-V4 and lead AVL (red arrows). Reciprocal ST segment depression is noted in the inferior leads, lead II and lead III (blue arrows) 
controlled, ongoing endothelial inflammation and antiretroviral drugs are known to contribute to an increased risk of atherosclerosis in HIV infection ${ }^{[6]}$.

In preparation for intervention, the patient received 2000 IU FVIII concentrate prior to percutaneous coronary intervention ( $\mathrm{PCl}$ ) to raise his FVIII level to $100 \%$. An angiogram was performed which revealed multiple vessel stenosis. This was a tight proximalvessel stenosis of the left anterior descending coronary artery (LAD) with TIMI 1 flow and expected poor filling distal of the stenosis. Additionally, the right coronary artery (RCA) showed intermediate mid-vessel stenosis. Primary $\mathrm{PCl}$ with stent insertion to the proximal LAD was performed. A $2.75 \mathrm{~mm} \times 16 \mathrm{~mm}$ resolute stent was inserted with a TIMI3 flow (complete filling of the distal vessel) confirmed post-stenting. Dual antiplatelet therapy comprising $300 \mathrm{mg}$ clopidogrel and $300 \mathrm{mg}$ aspirin was given orally. The patient stayed in hospital for a further five days post- $\mathrm{PCl}$, during which there were no significant bleeding complications. His FVIII

\begin{tabular}{l|l} 
DRUG & $\begin{array}{l}\text { DOSAGE PRESCRIBED AT } \\
\text { DISCHARGE }\end{array}$ \\
\hline Clopidogrel $^{\circledR}$ & $75 \mathrm{mg}$ daily p.o for 6 to 12 months \\
\hline Aspirin $^{\circledR}$ & $75 \mathrm{mg}$ daily p.o \\
\hline Simvastatin $^{\circledR}$ & $40 \mathrm{mg}$ daily p.o \\
\hline Carvedilol $^{\circledR}$ & $3.125 \mathrm{mg}$ twice daily p.o \\
\hline Enalapril $^{\circledR}$ & $2.5 \mathrm{mg}$ twice daily p.o \\
\hline Spironolactone $^{\circledR}$ & $12.5 \mathrm{mg}$ daily p.o \\
\hline Eloctate $(\mathrm{rFVIIIFC})^{\circledR}$ & $50 \mathrm{IU} / \mathrm{kg}$ intravenously every 3 days \\
\hline
\end{tabular}

Table 1. Medication list at discharge post- left anterior descending coronary artery (LAD) stenting

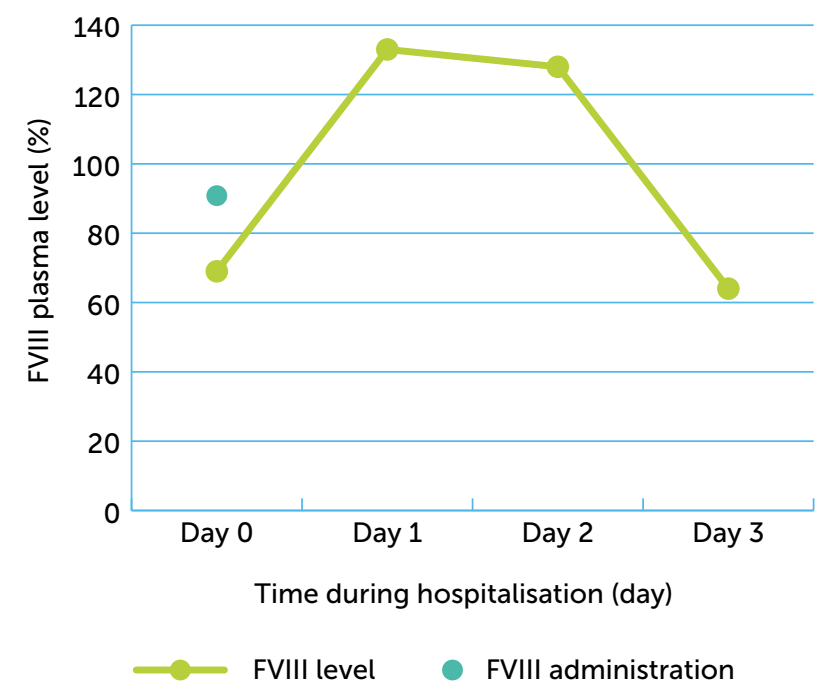

Figure 2: Factor level as a function of time during hospitalisation levels remained above $60 \mathrm{IU} / \mathrm{dL}$ until he went back to his regular prophylaxis regimen, which was not adjusted (see figure 2).

Three months after the STEMI, the patient underwent a right ankle fusion procedure for haemophilic arthropathy. He was still on extended halflife FVIII concentrate. No additional factor was required either during or post-procedure, and he did not present with significant blood loss.

One year post-PCI and LAD stenting, the patient remains on low dose $75 \mathrm{mg}$ aspirin and $150 \mathrm{mg}$ clopidogrel. The patient had received extended halflife FVIII concentrate as part of an extended study; due to the study ending and the product not being registered in South Africa, his haemophilia treatment has now changed back to normal plasma-derived FVIII replacement therapy. His joint bleeds have not increased and his spontaneous bleed rate remains at zero on prophylaxis. He has not experienced cardiac related symptoms to date.

\section{Discussion}

We believe this patient provides a number of lessons that can help improve our management of severe haemophilia A with STEMI. The patient was managed according to the European Society of Cardiology guidelines for the management of acute STEMI, although these guidelines are not specifically extended to people with bleeding diatheses such as haemophilia ${ }^{[7]}$. Other than in the immediate postoperative setting, clotting factor prophylaxis did not require adjustment, even with the addition of dual antiplatelet therapy. The patient remained bleed-free even with antiplatelet therapy on board; our clinical impression is that this confirms that intra-articular bleeds are not mediated through platelet dysfunction. The dose of antiplatelet therapy was adjusted to minimise the risk of bleeding but remained protective in keeping the stent patent ${ }^{[8]}$. This dose adjustment was based on clinical assessment and may require further assessment to establish the optimum dose in the context of haemophilia. While the current practice recommends FVIII levels of $30-80 \%$ in the postoperative period ${ }^{[9]}$, in this patient we kept the FVIII level post $\mathrm{PCl}$ above $50 \%$ to minimise the risk of bleeding due to the concurrent antiplatelet therapy.

\section{Conclusion}

PWH are at risk of developing atherosclerosis and associated cardiovascular disease equivalent to that seen in the general age-matched population. 
Management guidelines for PWH should include regular screening for risk factors associated with atherosclerosis. Any risk factor identified should be actively managed in specialised consultation as indicated ${ }^{[10,11]}$. Managing acute complications, such as myocardial infarction, is challenging in patients with an underlying bleeding diathesis. These patients are currently managed according to guidelines intended for the general population. Although replacement therapy allows the guidelines to be followed to some extent, definitive guidelines based on prospective studies are desperately needed in patients with a bleeding diathesis.

\section{Acknowledgements}

The authors have advised no interests that might be perceived as posing a conflict or bias.

Informed consent has been obtained from the participants in the study reported in this paper.

\section{References}

1. Angelini D, Konkle BA, Sood SL. Aging among persons with hemophilia: contemporary concerns. Semin Hematol 2016; 53(1): 35-9.

2. Biere-Rafi S, Baarslag MA, Peters M, Kruip MJ, et al. Cardiovascular risk assessment in haemophilia patients. Thromb Haemost 2011; 105(2): 274-8.
3. Kamphuisen PW, ten Cate H. Cardiovascular risk in patients with hemophilia. Blood 2014; 123(9): 1297-301.

4. Sousos N, Gavriilaki E, Vakalopoulou S, Garipidou V. Understanding cardiovascular risk in hemophilia: A step towards prevention and management. Thromb Res 2016; 140: 14-21.

5. Dolan $\mathrm{G}$. The challenge of an ageing haemophilic population. Haemophilia 2010; 16 Suppl 5: 11-16.

6. Cerrato E, Calcagno A, D'Ascenzo F, et al. Cardiovascular disease in HIV patients: from bench to bedside and backwards. Open Heart 2015; 2(1): e000174.

7. Ibanez B, James S, Agewall S, et al. 2017 ESC Guidelines for the management of acute myocardial infarction in patients presenting with ST-segment elevation: The Task Force for the management of acute myocardial infarction in patients presenting with ST-segment elevation of the European Society of Cardiology (ESC). Eur Heart J 2017; 39(2): 119-77. doi: 10.1093/eurheartj/ehx393.

8. Helft G. Dual antiplatelet therapy duration after drug-eluting stents: how long? J Thorac Dis 2016; 8(8): E844-6.

9. Schutgens RE, Tuinenburg A, Roosendaal G, Guyomi SH, Mauser-Bunschoten EP. Treatment of ischaemic heart disease in haemophilia patients: an institutional guideline. Haemophilia 2009; 15(4): 952-8.

10. Philipp $C$. The aging patient with hemophilia: complications, comorbidities, and management issues. Hematology Am Soc Hematol Educ Program 2010; 2010: 191-6.

11. Pocoski J, Ma A, Kessler CM, Boklage S, Humphries TJ. Cardiovascular comorbidities are increased in U.S. patients with haemophilia A: a retrospective database analysis. Haemophilia 2014; 20(4): 472-8.

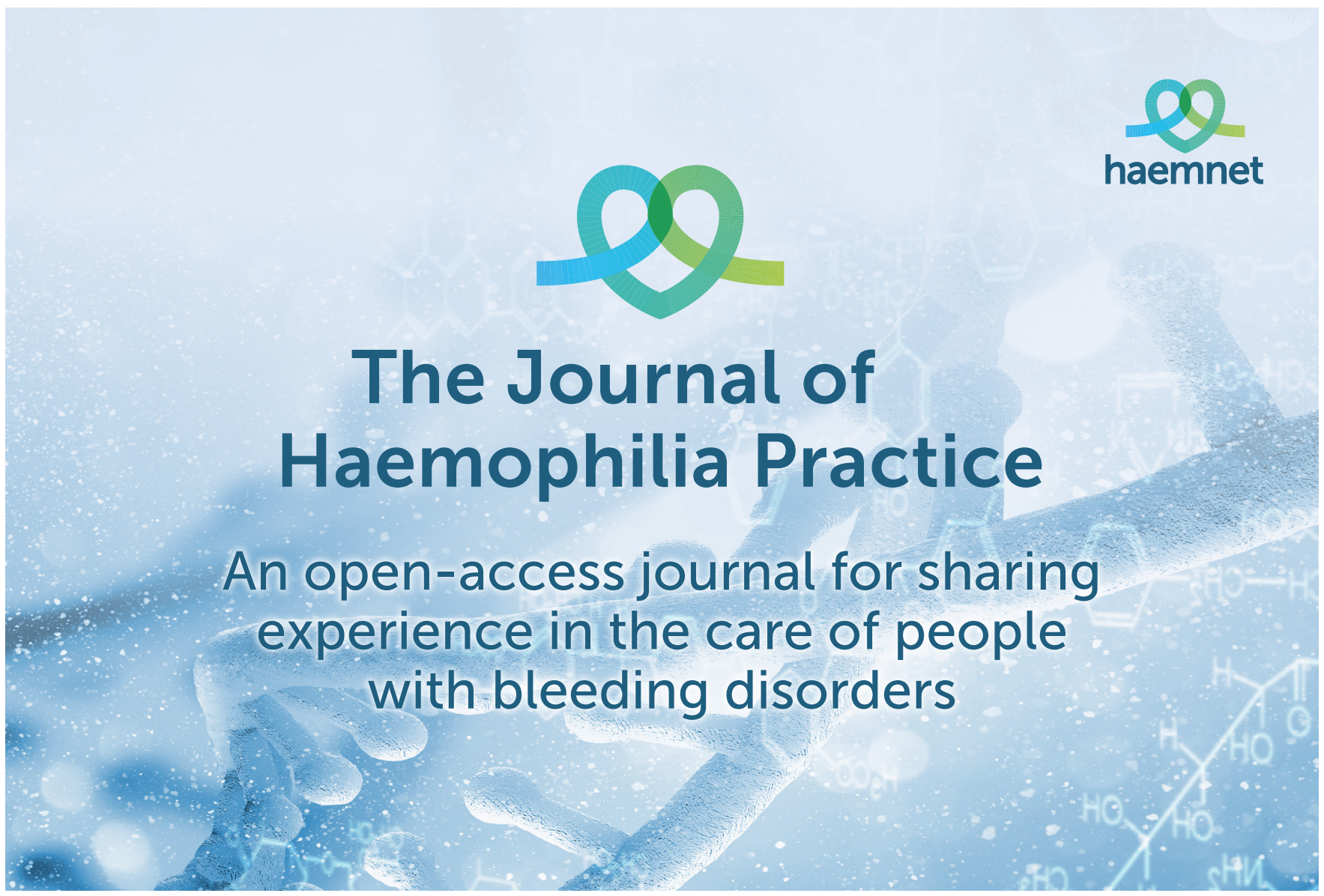

